typeset using JPSJ.sty $<$ ver.1.0b $>$

\title{
Theory of Tunneling Conductance for Normal Metal/Insulator/ Triplet Superconductor Junction
}

\author{
Masashi Yamashiro, Yukio Tanaka, Yasunari Tanuma and Satoshi Kashiwaya, \\ Graduate School of Science and Technology, Niigata University, Ikarashi, Niigata 950-2181 \\ ${ }^{1}$ Electrotechnical Laboratory, Tsukuba, Ibaraki 305-9568
}

(Received August 23, 2021)

\begin{abstract}
Tunneling conductance spectra of normal metal/insulator/triplet superconductor junctions are investigated theoretically. As triplet paring states we select several types of symmetries that are promising candidates for the superconducting states in $\mathrm{UPt}_{3}$ and in $\mathrm{Sr}_{2} \mathrm{RuO}_{4}$. The calculated conductance spectra are sensitive to the orientation of the junction which reflects the anisotropy of the pairing states. They show either zero-bias conductance peaks or gap-like structures depending on the orientation of the junctions. The existence of a residual density of states, peculiar to nonunitary states, is shown to have a significant influence on the properties of the conductance spectra. Present results serve as a guide for the experimental determination of the symmetry of the pair potentials in $\mathrm{UPt}_{3}$ and $\mathrm{Sr}_{2} \mathrm{RuO}_{4}$.
\end{abstract}

KEYWORDS: triplet superconductor, nonunitary pair potential, zero-bias conductance peak,

\section{$\S 1 . \quad$ Introduction}

Since the discovery of superconductivity in heavy fermion compounds the determination of the symmetry of the pair potential in these materials has been an important issue.(1) Among the heavy fermion superconductors, so far properties of $\mathrm{UPt}_{3}$ have been studied most extensively. Based on NMR experiments the possibility of odd parity pairing states, i.e., triplet pairing states, has been suggested. Theoretically, several papers propose two-dimensional even parity states, i.e. singlet pairing states, 目国) while others suggest triplet pairing states belonging to the one-

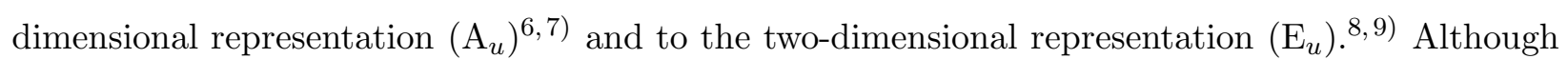

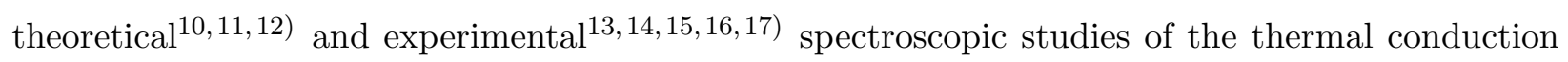
and transverse sound attenuation of $\mathrm{UPt}_{3}$ have been performed, the symmetry of the pair potential could not yet been determined.

Recently superconductivity has been discovered in $\mathrm{Sr}_{2} \mathrm{RuO}_{4}, 18$ ) which is the first example of a noncuprate layered perovskite superconductor. Since this compound is isostructual to the cuprate superconductors the electronic properties in the normal state 19 ) and superconducting state29) are 
highly anisotropic. Several experiments2,22) indicate a large residual density of states of quasiparticles at low temperatures. In addition there is evidence for the existence of ferromagnetic spin fluctuations.23.24) Based on these facts two-dimensional triplet superconducting states, belonging to the two-dimensional $\mathrm{E}_{u}$ symmetry, have been proposed for $\mathrm{Sr}_{2} \mathrm{RuO}_{4}$. 25, 26) Similar to the case of $\mathrm{UPt}_{3}$ the symmetry of the pair potential of $\mathrm{Sr}_{2} \mathrm{RuO}_{4}$ has not yet been determined.

Phase-sensitive measurements provide the most useful information for the determination of the symmetry of the pair potentials.27) Recently it was shown that tunneling spectroscopy has the ability to detect the phase of the pair potential 28, 29, 30 as follows: In anisotropic superconductors quasiparticles feel different signs of the pair potentials depending on the directions of their motions.31) At the normal metal/superconductor interface the anisotropy of the pair potential significantly influences the properties of the Andreev reflections.32.33) As a result tunneling conductance spectra of the normal metal/insulator/anisotropic superconductor junctions are modified due to the anisotropy of pair potential 28,29) The most remarkable feature is the existence of zerobias conductance peaks (ZBCP) in the tunneling spectra for $d$-wave symmetry. The origin of these peaks is the localized zero energy states (ZES) 34 due to the change of sign of the pair potential in k-space.30) ZBCP have actually been observed in experiments on high- $T_{c}$ superconductor,29.35,36) and the consistency between theory and experiments has been checked. (37, B8

To determine the symmetries of the pair potential of $\mathrm{UPt}_{3}$ and $\mathrm{Sr}_{2} \mathrm{RuO}_{4}$ it is meaningful to apply the phase sensitive capability of tunneling spectroscopy. For superconducting $\mathrm{UPt}_{3}$ various

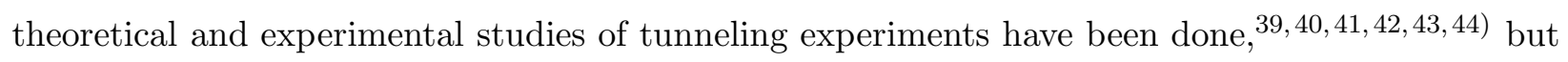
unfortunately effects of the phase have not been considered in these papers. Similar to the singlet cases, the appearance of ZBCP in conductance spectra is expected due to the change of sign of the pair potential. To apply the concepts of phase sensitivity to triplet superconductors additional studies are required, due to the large difference in the pairing states.

In the present paper, we investigate the tunneling conductance spectra of normal metal / insulator/ triplet superconductor $(N / I / T S)$ junctions by extending the previous theory for anisotropic singlet superconductors. Although there are several works concerning surface bound states in triplet superconductors, 国, (4) systematic studies for tunnel conductance in $N / I / T S$ junctions have not been performed. The following points are of interests: i) the orientations of ZBCP which gives essential information about the symmetries, ii) the spin dependence of the conductance spectra and iii) the influence of the nonunitary states. The organization of this paper is as follows: In Sec.2 a general formula of conductance spectroscopy for triplet superconductors is presented. Since it is meaningless to present calculations for all possible triplet symmetries, the results are given for several typical cases; promising pairing states for $\mathrm{UPt}_{3}$ in Sec. 3.1 and for $\mathrm{Sr}_{2} \mathrm{RuO}_{4}$ in Sec. 3.2. Finally in Sec.⿴囗十 we summarize our results and discuss future problems. 


\section{$\S 2 . \quad$ Conductance formula}

For the calculation, we assume three-dimensional $N / I / T S$ junctions with semi-infinite double layer structures in the clean limit. The flat interface is perpendicular to the $x$-axis, and is located at $x=0$ [Fig.1(a)]. The barrier potential at the interface has a delta-functional form $H \delta(x)$, where $\delta(x)$ and $H$ are the delta function and its amplitude, respectively. We also consider another orientation of the junction where the flat interface is perpendicular to the $z$-axis and located at $z=0$ [Fig.1(b)]. The Fermi wave number $k_{F}$ and the effective mass 39 , 47) $m$ are assumed to be equal both in the normal metal and in the superconductor. For simplicity we assume in the following calculations that the pair potentials are spatially constant. The wave function describing the quasiparticles in inhomogeneous anisotropic superconductors $\Psi(\boldsymbol{r})$ is obtained by the solutions of the Bogoliubov-de Gennes (BdG) equation. 31, 34) After applying the quasi-classical approximation, BdG equations are reduced to the Andreev equation, $11,34,48$

$$
\begin{gathered}
E u_{s}(\boldsymbol{r})=-i v_{F} \boldsymbol{k} \cdot \nabla u_{s}(\boldsymbol{r})+\sum_{s^{\prime}} \Delta_{s s^{\prime}}(\theta, \phi) v_{s^{\prime}}(\boldsymbol{r}) \\
E v_{s}(\boldsymbol{r})=i v_{F} \boldsymbol{k} \cdot \nabla v_{s}(\boldsymbol{r})+\sum_{s^{\prime}} \Delta_{s s^{\prime}}^{*}(\theta, \phi) u_{s^{\prime}}(\boldsymbol{r}) .
\end{gathered}
$$

The quantities $u_{s}(\boldsymbol{r})$ and $v_{s}(\boldsymbol{r})$ are electronlike quasiparticles (ELQ) and holelike quasiparticles (HLQ), respectively with spin index $s=\uparrow$ or $s=\downarrow$. In Eq. (2.1), $\boldsymbol{k}$ describes the relative motion of the Cooper pairs fixed on the Fermi surface $\left(|\boldsymbol{k}|=k_{F}\right), \boldsymbol{r}$ the center of mass coordinates of the pair potential and $v_{F}$ the Fermi velocity. The wave functions $u_{s}(\boldsymbol{r})$ and $v_{s}(\boldsymbol{r})$ are obtained by

neglecting the fast oscillating plane-wave part, according to the quasiclassical approximations. (31, (4) In this approximation, we assume that the effective pair potential is given by

$$
\begin{aligned}
& \boldsymbol{\Delta}(\boldsymbol{k}, \boldsymbol{r})=\boldsymbol{\Delta}(\theta, \phi) \Theta(x), \quad z \text {-y plane interface } \\
&=\boldsymbol{\Delta}(\theta, \phi) \Theta(z), \quad x-y \text { plane interface } \\
& \frac{k_{x}+i k_{y}}{|\boldsymbol{k}|}=e^{i \phi} \sin \theta, \quad \frac{k_{z}}{|\boldsymbol{k}|}=\cos \theta
\end{aligned}
$$

where $\theta$ is the polar angle and $\phi$ is the azimuthal angle in the $x-y$ plane. The quantities $\Theta(x)$ and $\Theta(z)$ are the Heaviside step function. The pair potential matrix is represented as

$$
\boldsymbol{\Delta}(\theta, \phi)=\left(\begin{array}{cc}
\Delta_{\uparrow \uparrow}(\theta, \phi) & \Delta_{\uparrow \downarrow}(\theta, \phi) \\
\Delta_{\downarrow \uparrow}(\theta, \phi) & \Delta_{\downarrow \downarrow}(\theta, \phi)
\end{array}\right) .
$$

In the present paper, we neglect the spin-orbit coupling of the quasiparticles. A formula for the tunneling conductance of the $N / I / T S$ junction is derived from the reflection and transmission probabilities of the electrons injected from the normal metal with angles $\theta$ and $\phi$. In the following, we assume that an electron is injected with an equal probability for up and down spin. The 
injected electron is reflected as an electron (normal reflection) and as a hole (Andreev reflection). When the interface is perpendicular to the $x$-axis ( $z$-y plane interface) [Fig.1(a)], the transmitted ELQ and HLQ feel different effective pair potentials $\Delta_{s s^{\prime}}\left(\theta, \phi_{+}\right)$and $\Delta_{s s^{\prime}}\left(\theta, \phi_{-}\right)$, with $\phi_{+}=\phi$ and $\phi_{-}=\pi-\phi$. On the other hand, in the case when the interface is perpendicular to the $z$-axis ( $x$ - $y$ plane interface), two kinds of quasiparticles feel $\Delta_{s s^{\prime}}\left(\theta_{+}, \phi\right)$ and $\Delta_{s s^{\prime}}\left(\theta_{-}, \phi\right)$, with $\theta_{+}=\theta$ and $\theta_{-}=\pi-\theta$ [Fig.1(b)], respectively. The coefficients of the Andreev reflection $a_{s s^{\prime}}(E, \theta, \phi)$ and the normal reflection $b_{s s^{\prime}}(E, \theta, \phi)$ are determined by solving the BdG equation under the following boundary conditions

$$
\left.\Psi(\boldsymbol{r})\right|_{x=0_{-}}=\left.\Psi(\boldsymbol{r})\right|_{x=0_{+}},\left.\quad \frac{d \Psi(\boldsymbol{r})}{d x}\right|_{x=0_{-}}=\left.\frac{d \Psi(\boldsymbol{r})}{d x}\right|_{x=0_{+}}-\left.\frac{2 m H}{\hbar^{2}} \Psi(\boldsymbol{r})\right|_{x=0_{-}}
$$

for $z-y$ plane interface and

$$
\left.\Psi(\boldsymbol{r})\right|_{z=0_{-}}=\left.\Psi(\boldsymbol{r})\right|_{z=0_{+}},\left.\quad \frac{d \Psi(\boldsymbol{r})}{d z}\right|_{z=0_{-}}=\left.\frac{d \Psi(\boldsymbol{r})}{d z}\right|_{z=0_{+}}-\left.\frac{2 m H}{\hbar^{2}} \Psi(\boldsymbol{r})\right|_{z=0_{-}}
$$

for $x-y$ plane interface. Using the obtained coefficients, the normalized tunneling conductance at zero temperature is calculated according to the formula given in our previous work 28, 30

$$
\begin{aligned}
\sigma(E)= & \frac{\int_{0}^{\pi} \int_{-\pi / 2}^{\pi / 2}\left[\sigma_{S, \uparrow}(E, \theta, \phi)+\sigma_{S, \downarrow}(E, \theta, \phi)\right] \sigma_{N}(\theta, \phi) \sin ^{2} \theta \cos \phi d \theta d \phi}{\int_{0}^{\pi} \int_{-\pi / 2}^{\pi / 2} 2 \sigma_{N}(\theta, \phi) \sin ^{2} \theta \cos \phi d \theta d \phi}, \quad z \text {-y plane interface } \\
& =\frac{\int_{0}^{\pi / 2} \int_{0}^{2 \pi}\left[\sigma_{S, \uparrow}(E, \theta, \phi)+\sigma_{S, \downarrow}(E, \theta, \phi)\right] \sigma_{N}(\theta, \phi) \sin \theta \cos \theta d \theta d \phi}{\int_{0}^{\pi / 2} \int_{0}^{2 \pi} 2 \sigma_{N}(\theta, \phi) \sin \theta \cos \theta d \theta d \phi}, \quad x-y \text { plane interface }
\end{aligned}
$$

where $\sigma_{N}(\theta, \phi)$ denotes the normal state tunneling conductance given by

$$
\begin{aligned}
\sigma_{N}(\theta, \phi)= & \frac{\sin ^{2} \theta \cos ^{2} \phi}{\sin ^{2} \theta \cos ^{2} \phi+Z^{2}}, \quad z-y \text { plane interface } \\
= & \frac{\cos ^{2} \theta}{\cos ^{2} \theta+Z^{2}}, \quad x-y \text { plane interface, } \\
& Z=\frac{m H}{\hbar^{2} k_{F}}
\end{aligned}
$$

Here $Z$ and $E$ denote an effective barrier parameter and an energy of quasi-particles measured from the Fermi energy, respectively. The quantity $\sigma_{S, s}(E, \theta, \phi)$, which is the normalized conductance for the specific spin component $(s=\uparrow$ or $\downarrow$ ) for fixed $\theta$ and $\phi$, is given as

$$
\sigma_{S, s}(E, \theta, \phi)=\frac{1+\left|a_{\uparrow s}\right|^{2}+\left|a_{\downarrow s}\right|^{2}-\left|b_{\uparrow s}\right|^{2}-\left|b_{\downarrow s}\right|^{2}}{\sigma_{N}(\theta, \phi)},
$$

using the normal and Andreev reflection coefficients $b_{s s^{\prime}}(E, \theta, \phi)$ and $a_{s s^{\prime}}(E, \theta, \phi)$, respectively $(s=\uparrow, \downarrow)$. Eqs.(2.6) and (2.8) obtained for the conductance are generic. Now it is straightforward to obtain the tunneling conductance spectra for any triplet superconductor when the irreducible representations of the pair potentials are given. For the following discussion, we point out that the low transparency limit of the junction means $\sigma_{N}(\theta, \phi) \rightarrow 0$, i.e., $Z \rightarrow \infty$. 


\section{$\S 3 . \quad$ Tunneling spectra in triplet superconductors}

\section{$3.1 \mathrm{UPt}_{3}$}

In this section, the tunneling conductance of a normal metal / insulator / $\mathrm{UPt}_{3}$ junction is calculated for several promising pairing states with hexagonal symmetry. There are one-dimensional (6) $\left(\mathrm{A}_{1 u}, \mathrm{~A}_{2 u}\right)$ and two-dimensiona 8 国) $\left(\mathrm{E}_{1 u}, \mathrm{E}_{2 u}\right)$ representations with triplet pairing states and a two-dimensional $\mathrm{E}_{1 g}$ state细国) with singlet pairing. A brief report of this section was already

given elsewhere.49) In the case of a one-dimensional representation, both states, $\mathrm{A}_{1 u}$ and $\mathrm{A}_{2 u}$, are nonunitary pair potentials. For both representations the normalized conductance $\sigma_{S, \uparrow}(E, \theta, \phi)$ is given as

$$
\begin{aligned}
\sigma_{S, \uparrow}(E, \theta, \phi) & =\frac{1+\sigma_{N}(\theta, \phi)|\Gamma|^{2}+\left[\sigma_{N}(\theta, \phi)-1\right]|\Gamma|^{4}}{\left|1+\left[\sigma_{N}(\theta, \phi)-1\right] \Gamma^{2}\right|^{2}}, \quad z \text {-y plane interface } \\
& =\frac{1+\sigma_{N}(\theta, \phi)|\Gamma|^{2}+\left[\sigma_{N}(\theta, \phi)-1\right]|\Gamma|^{4}}{\left|1-\left[\sigma_{N}(\theta, \phi)-1\right] \Gamma^{2}\right|^{2}}, \quad x \text {-y plane interface } \\
\Gamma & =\frac{E-\Omega}{\left|\Delta_{\uparrow \uparrow}(\theta, \phi)\right|}, \quad \Omega=\sqrt{E^{2}-\left|\Delta_{\uparrow \uparrow}(\theta, \phi)\right|^{2}}
\end{aligned}
$$

where $\Delta_{\uparrow \uparrow}(\theta, \phi)$ is given by

$\mathrm{A}_{1 u}$ :

$$
\Delta_{\uparrow \uparrow}(\theta, \phi)=\Delta_{0} \cos \theta, \Delta_{\uparrow \downarrow}(\theta, \phi)=\Delta_{\downarrow \uparrow}(\theta, \phi)=\Delta_{\downarrow \downarrow}(\theta, \phi)=0
$$

$\mathrm{A}_{2 u}$ :

$$
\Delta_{\uparrow \uparrow}(\theta, \phi)=\Delta_{0} \cos \theta \sin ^{2} \theta, \Delta_{\uparrow \downarrow}(\theta, \phi)=\Delta_{\downarrow \uparrow}(\theta, \phi)=\Delta_{\downarrow \downarrow}(\theta, \phi)=0 .
$$

$\sigma_{S, \downarrow}(E, \theta, \phi)$ is equal to unity due to the absence of the effective pair potentials. For the $z$ - $y$ plane interface junction $\sigma(E)$ shows a maximum at $E \sim 0.8 \Delta_{0}$ and at $E \sim 0.4 \Delta_{0}$ for the $A_{1 u}$ and the $\mathrm{A}_{2 u}$ states, respectively[Fig.2]. For the two cases the conductance has a different peak position due to the existence of a $\sin ^{2} \theta$ factor. Reflecting the residual density of states of quasiparticles with down spin, $\sigma(0)$ converges not to 0 , but to 0.5 for the $z$-y plane interface for $Z \rightarrow \infty$. For this case we can show that $\sigma(E)$ corresponds to the bulk density of states. For a $x-y$ plane interface junction, $\sigma(0)$ increases monotonically with increasing $Z$ for both, the $\mathrm{A}_{1 u}$ and the $\mathrm{A}_{2 u}$ states. In this case, quasiparticles form ZES on a finite range of the Fermi surface (see Table I).

The tunneling conductance $\sigma_{S, \downarrow}(E, \theta, \phi)$ of the $\mathrm{E}_{1 u}$ state is given as

$$
\begin{aligned}
\sigma_{S, \downarrow}(E, \theta, \phi) & =\frac{1+\sigma_{N}(\theta, \phi)|\Gamma|^{2}+\left[\sigma_{N}(\theta, \phi)-1\right]|\Gamma|^{4}}{\left|1+e^{-4 i \phi}\left[\sigma_{N}(\theta, \phi)-1\right] \Gamma^{2}\right|^{2}}, \quad z \text {-y plane interface } \\
& =\frac{1+\sigma_{N}(\theta, \phi)|\Gamma|^{2}+\left[\sigma_{N}(\theta, \phi)-1\right]|\Gamma|^{4}}{\left|1-\left[\sigma_{N}(\theta, \phi)-1\right] \Gamma^{2}\right|^{2}}, \quad x \text {-y plane interface } \\
\Gamma & =\frac{E-\Omega}{\left|\Delta_{\downarrow \downarrow}(\theta, \phi)\right|}, \quad \Omega=\sqrt{E^{2}-\left|\Delta_{\downarrow \downarrow}(\theta, \phi)\right|^{2}} .
\end{aligned}
$$




$$
\Delta_{\downarrow \downarrow}(\theta, \phi)=\Delta_{0} e^{2 i \phi} \cos \theta \sin ^{2} \theta, \Delta_{\uparrow \downarrow}(\theta, \phi)=\Delta_{\downarrow \uparrow}(\theta, \phi)=\Delta_{\uparrow \uparrow}(\theta, \phi)=0
$$

$\sigma_{S, \uparrow}(E, \theta, \phi)$ is equal to unity due to the absence of other pair potentials except for $\Delta_{\downarrow}(\theta, \phi)$. The injected quasiparticles with down spin form ZES at the interface depending on the direction of their motions for $\sigma_{N}(\theta, \phi) \rightarrow 0$. For $z$-y plane interface junctions, ZES is expected on the line, $\phi= \pm \pi / 4$, whereas for a $x-y$ plane interface junction, ZES is expected on a finite area of the Fermi surface, $0<\theta<\pi / 2$ [Fig.3].

The $\mathrm{E}_{2 u}$ representation is a unitary state and $\sigma(E)$ is given as

$$
\begin{gathered}
\sigma_{S, \downarrow}(E, \theta, \phi)=\sigma_{S, \uparrow}(E, \theta, \phi)=\frac{1+\sigma_{N}(\theta, \phi)|\Gamma|^{2}+\left[\sigma_{N}(\theta, \phi)-1\right]|\Gamma|^{4}}{\left|1+e^{-4 i \phi}\left[\sigma_{N}(\theta, \phi)-1\right] \Gamma^{2}\right|^{2}}, \quad z \text {-y plane interface } \\
=\frac{1+\sigma_{N}(\theta, \phi)|\Gamma|^{2}+\left[\sigma_{N}(\theta, \phi)-1\right]|\Gamma|^{4}}{\left|1-\left[\sigma_{N}(\theta, \phi)-1\right] \Gamma^{2}\right|^{2}}, \quad x \text {-y plane interface } \\
\Gamma=\frac{E-\Omega}{\left|\Delta_{\uparrow \downarrow}(\theta, \phi)\right|}, \quad \Omega=\sqrt{E^{2}-\left|\Delta_{\uparrow \downarrow}(\theta, \phi)\right|^{2}} . \\
\Delta_{\uparrow \downarrow}(\theta, \phi)=\Delta_{\downarrow \uparrow}(\theta, \phi)=\Delta_{0} e^{2 i \phi} \cos \theta \sin ^{2} \theta, \quad \Delta_{\uparrow \uparrow}(\theta, \phi)=\Delta_{\downarrow \downarrow}(\theta, \phi)=0 .
\end{gathered}
$$

The line shape of $\sigma(E)$ is similar to that of the $\mathrm{E}_{1 u}$ state since both pair potentials have the same orbital dependence. However, $\sigma(E)$ for the $\mathrm{E}_{1 u}$ state is always larger than 0.5 due to the nonunitarity[Fig.3].

Finally, let us consider another promising state, i.e., the $\mathrm{E}_{1 g}$ state, where the pair potential is a singlet. Applying the previous theory by Tanaka and Kashiwaya, 28, 29, 30,50 $\sigma_{S, \uparrow}(E, \theta, \phi)$ and $\sigma_{S, \downarrow}(E, \theta, \phi)$ are obtained as

$$
\begin{gathered}
\sigma_{S, \uparrow}(E, \theta, \phi)=\sigma_{S, \downarrow}(E, \theta, \phi)=\frac{1+\sigma_{N}(\theta, \phi)|\Gamma|^{2}+\left[\sigma_{N}(\theta, \phi)-1\right]|\Gamma|^{4}}{\left|1-e^{-2 i \phi}\left[\sigma_{N}(\theta, \phi)-1\right] \Gamma^{2}\right|^{2}}, \quad z-y \text { plane interface } \\
=\frac{1+\sigma_{N}(\theta, \phi)|\Gamma|^{2}+\left[\sigma_{N}(\theta, \phi)-1\right]|\Gamma|^{4}}{\left|1-\left[\sigma_{N}(\theta, \phi)-1\right] \Gamma^{2}\right|^{2}}, \quad x-y \text { plane interface } \\
\Gamma=\frac{E-\Omega}{|\Delta(\theta, \phi)|}, \quad \Omega=\sqrt{E^{2}-|\Delta(\theta, \phi)|^{2}}
\end{gathered}
$$

with $\Delta(\theta, \phi)=\Delta_{0} e^{2 i \phi} \cos \theta \sin \theta$. For $z$-y plane interface junctions, ZES is expected on the line, $\phi=0$, whereas for a $x-y$ plane interface junction, ZES is expected on a finite area of the Fermi surface, $0<\theta<\pi / 2$. [Fig.4].

All pair potentials discussed in this section are promising candidates for the superconducting state in $\mathrm{UPt}_{3}$. For all of them ZBCP appear when the interface is perpendicular to the $z$-axis. Performing tunneling spectroscopy measurements on a high purity $\mathrm{UPt}_{3}$ sample with a well oriented junction, ZBCP are expected to be observed for the junction with a $x-y$ plane interface. We strongly hope that ZBCP will be observed in the actual experiments in near future. For the case of a $z-y$ plane interface, a wide variety of line shapes of the conductance are expected, because of the irreducible representations of the pair potentials and the direction of the junctions. 


\section{2 $\mathrm{Sr}_{2} \mathrm{RuO}_{4}$}

As a first case of this section, we consider two nonunitary pair potentials. Previously, we have

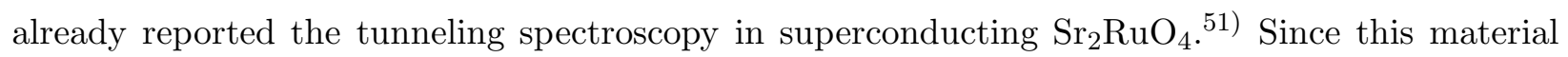
has two-dimensional electronic properties, we used a nearly cylindrical Fermi surface. The resultant conductance formula (2.6) is reduced to Eqs.(4) to (6) in Ref. 51. In this previous paper we chose two kinds of nonunitary pair potentials with tetragonal symmetry, $\Delta_{\uparrow \uparrow}(\theta, \phi)=\Delta_{0} \sin \theta(\sin \phi+\cos \phi)$ $\left(\mathrm{E}_{u}(1)\right.$ state) and $\Delta_{\uparrow \uparrow}(\theta, \phi)=\Delta_{0} e^{i \phi} \sin \theta\left(\mathrm{E}_{u}(2)\right.$ state). The obtained conductance formulas were given by Eqs.(7) to (10) and spectra were shown in Figs. 2 and 3 in Ref. 51 . In a $x$-y plane interface junction, $\sigma(E)$ coincides with the bulk density of states for both, the $\mathrm{E}_{u}(1)$ and the $\mathrm{E}_{u}(2)$ states. In the $\mathrm{E}_{u}(2)$ case, U-shaped spectra are obtained [Fig.3 in Ref. 51] due to the nearly twodimensionality of the system. In the case of a $z-y$ plane interface junction with low transparency, ZES is expected for $-\pi / 4 \leq \phi \leq \pi / 4$ for the $\mathrm{E}_{u}(1)$ state, while in the case of the $\mathrm{E}_{u}(2)$ state, ZES is expected for $\phi=0$. Consequently, $\sigma(0)$ of $\mathrm{E}_{u}(1)$ diverges with increasing $Z$, while $\sigma(0)$ of the $\mathrm{E}_{u}(2)$ converges to a finite value. Our results are consistent with recent results by Honerkamp et al.22 As a second case, we calculate tunneling conductance spectra for $\mathrm{Sr}_{2} \mathrm{RuO}_{4}$ with unitary pair potentials which are given as $\Delta_{\uparrow \downarrow}=\Delta_{0} \sin \theta(\sin \phi+\cos \phi)\left(\mathrm{E}_{u}(\mathrm{U} 1)\right.$ state $)$ and $\Delta_{\uparrow \downarrow}=\Delta_{0} e^{i \phi} \sin \theta$ $\left(\mathrm{E}_{u}(\mathrm{U} 2)\right.$ state). For both states, $\Delta_{\uparrow \uparrow}$ and $\Delta_{\downarrow \downarrow}=0$ and $\Delta_{\uparrow \downarrow}=\Delta_{\downarrow \uparrow}$ are satisfied. A conductance formulas for these pair potentials are given as

$\mathrm{E}_{u}(\mathrm{U} 1)$ :

$$
\begin{aligned}
& \sigma_{S, \uparrow}(E, \theta, \phi)=\sigma_{S, \downarrow}(E, \theta, \phi) \\
&= \frac{1+\sigma_{N}(\theta, \phi)\left|\Gamma_{+}\right|^{2}+\left[\sigma_{N}(\theta, \phi)-1\right]\left|\Gamma_{+}\right|^{2}\left|\Gamma_{-}\right|^{2}}{\left|1+\left[\sigma_{N}(\theta, \phi)-1\right] \Gamma_{+} \Gamma_{-}\right|^{2}}, \quad z \text {-y plane interface } \\
&=\frac{1+\sigma_{N}(\theta, \phi)\left|\Gamma_{+}\right|^{2}+\left[\sigma_{N}(\theta, \phi)-1\right]\left|\Gamma_{+}\right|^{4}}{\left|1+\left[\sigma_{N}(\theta, \phi)-1\right] \Gamma_{+}^{2}\right|^{2}}, \quad x \text {-y plane interface } \\
& \Gamma_{ \pm}=\frac{\Delta_{0} \sin \theta(\sin \phi \pm \cos \phi)}{E+\Omega_{ \pm}}, \quad \Omega_{ \pm}=\sqrt{E^{2}-\left|\Delta_{0} \sin \theta(\sin \phi \pm \cos \phi)\right|^{2}} .
\end{aligned}
$$

$\mathrm{E}_{u}(\mathrm{U} 2)$ :

$$
\begin{aligned}
\sigma_{S, \uparrow}(E, \theta, \phi)= & \sigma_{S, \downarrow}(E, \theta, \phi) \\
= & \frac{1+\sigma_{N}(\theta, \phi)|\Gamma|^{2}+\left[\sigma_{N}(\theta, \phi)-1\right]|\Gamma|^{4}}{\left|1-e^{-2 i \phi}\left[\sigma_{N}(\theta, \phi)-1\right] \Gamma^{2}\right|^{2}}, \quad z \text {-y plane interface } \\
= & \frac{1+\sigma_{N}(\theta, \phi)|\Gamma|^{2}+\left[\sigma_{N}(\theta, \phi)-1\right]|\Gamma|^{4}}{\left|1+\left[\sigma_{N}(\theta, \phi)-1\right] \Gamma^{2}\right|^{2}}, \quad x \text {-y plane interface } \\
& \Gamma=\frac{E-\Omega}{\left|\Delta_{\uparrow \uparrow}(\theta, \phi)\right|}, \quad \Omega=\sqrt{E^{2}-\left|\Delta_{\uparrow \uparrow}(\theta, \phi)\right|^{2}} .
\end{aligned}
$$


The obtained spectra are similar to those of the $\mathrm{E}_{u}(1)$ and $\mathrm{E}_{u}(2)$ cases as shown in Fig.5. However, $\sigma(0)$ vanishes with the increasing $Z$ in the case of $x-y$ plane interface due to the absence of residual density of states of quasiparticles.

Throughout this paper the $\delta$-function model has been used to express the insulating barrier. For the junctions of singlet superconductors we have already confirmed that if the finite thickness of the insulating barrier is taken into account the essential results of the $\delta$-function model, e.g., the conditions of the existence of the zero energy states, are not changed at all.28, 53) For the junctions of triplet superconductors it is interesting to calculate the tunneling conductance in a model where the thickness of the insulating region is given as $d_{i}$. Then only the tunneling conductance in the normal state is modified to

$$
\begin{gathered}
\sigma_{N}(\theta, \phi)=\frac{4 Z^{2}(\theta, \phi)}{\left[1-Z^{2}(\theta, \phi)\right]^{2} \sinh ^{2}\left(\lambda d_{i}\right)+4 Z^{2}(\theta, \phi) \cosh ^{2}\left(\lambda d_{i}\right)}, \\
\lambda=\left[1-\kappa^{2} \alpha^{2}(\theta, \phi)\right]^{\frac{1}{2}} \lambda_{0}, \quad Z(\theta, \phi)=\frac{\kappa \alpha(\theta, \phi)}{\sqrt{1-\kappa^{2} \alpha^{2}(\theta, \phi)}} \\
\lambda_{0}=\sqrt{\frac{2 m U_{0}}{\hbar}}, \quad \kappa=\frac{k_{F}}{\lambda_{0}} .
\end{gathered}
$$

In the above, $U_{0}$ is the magnitude of the Hartree potential in the insulating region. The form factors $\alpha(\theta, \phi)$ are given as $\alpha(\theta, \phi)=\sin \theta \cos \phi$ for the $z$-y plane interface and $\alpha(\theta, \phi)=\cos \theta$ for the $x-y$ plane interface. The resulting tunneling spectra for this normal conductance are shown in Fig.6 for the examples of $\mathrm{E}_{u}(1)$ and $\mathrm{E}_{u}(2)$. The obtained line shapes of the tunneling conductance are almost similar to those in Figs. 2 and 3 in Ref. 51. The increase of the thickness of the insulating barrier corresponds to that of $Z$ in the $\delta$-function model.

\section{$\S 4$. Conclusions}

The tunneling conductance spectra in normal metal/insulator/triplet superconductor junctions have been presented for various promising pairing states for the superconductors $\mathrm{UPt}_{3}$ and $\mathrm{Sr}_{2} \mathrm{RuO}_{4}$. The obtained conductance spectra exhibit very fruitful features, which in the low transparency limit can be classified into six cases:

(1) The first case is that $\sigma(0)$ monotonically increases with increasing $Z$. ZES is formed in a finite area on the Fermi surface. $\left(\mathrm{E}_{2 u}\right.$ state with a $x-y$ plane interface, $\mathrm{E}_{1 g}$ state with a $x-y$ plane interface, $\mathrm{E}_{u}(\mathrm{U} 1)$ state with a $z-y$ plane interface)

(2) The second case is that $\sigma(0)$ converges to zero for sufficiently large $Z$. ZES does not appear on the Fermi surface. ( $\mathrm{E}_{u}(\mathrm{U} 1)$ state with a $x-y$ plane interface, $\mathrm{E}_{u}(\mathrm{U} 2)$ state with a $x-y$ plane interface)

(3) The third case is that $\sigma(0)$ converges to nonzero value with increasing $Z$. Here ZES is formed on a certain line on the Fermi surface. ( $\mathrm{E}_{2 u}$ state with a $z-y$ plane interface, $\mathrm{E}_{1 g}$ state with a $z-y$ plane interface, $\mathrm{E}_{u}(\mathrm{U} 2)$ state with a $z-y$ plane interface) 
(4) The fourth case are nonunitary states with ZBCP. $\left(\mathrm{A}_{1 u}\right.$ state with a $x$-y plane interface, $\mathrm{A}_{2 u}$ state with a $x-y$ plane interface, $\mathrm{E}_{1 u}$ state with a $x$-y plane interface, $\mathrm{E}_{u}(1)$ state with a $z$ - $y$ plane interface)

(5) The fifth case are nonunitary states where for sufficiently larger $Z, \sigma(0)$ converges to 0.5 . $\left(\mathrm{A}_{1 u}\right.$ state with a $z-y$ plane interface, $\mathrm{A}_{2 u}$ state with a $z-y$ plane interface, $\mathrm{E}_{u}(1)$ state with a $x-y$ plane interface, $\mathrm{E}_{u}(2)$ state with a $x-y$ plane interface)

(6) The sixth case are nonunitary states where $\sigma(E)$ converges to a certain finite value larger than 0.5. ( $\mathrm{E}_{1 u}$ state a with $z$-y plane interface, $\mathrm{E}_{u}(2)$ state with a $z$-y plane interface)

The regions of the Fermi surface which contribute to the ZES is summarized in Tables I to II. Our classification can serve as a guide in the determination of the symmetry of the pair potentials in $\mathrm{UPt}_{3}$ and $\mathrm{Sr}_{2} \mathrm{RuO}_{4}$. We hope that tunneling spectroscopy experiments will be performed on a very clean sample using a well oriented junction with flat interfaces. In the determination of the symmetry of the unknown pair potentials the barrier height and the directions of the tunneling conductance are the most important points.

Finally, we comment on the gauge invariance concerning the condition of ZES. For this purpose, let us discuss the role of external phase $\chi_{0}$ and internal phase $\varphi^{i}$ in anisotropic superconductors. In general, pair potential $\Delta_{s s^{\prime}}(\theta, \phi)$ can be expressed as $\Delta_{s s^{\prime}}(\theta, \phi)=\left|\Delta_{s s^{\prime}}(\theta, \phi)\right| \exp \left[i\left(\varphi^{i}+\chi_{0}\right)\right]$. The internal phase $\varphi^{i}$ of the pair potential is measured from the $c$ - and $a$-axes using polar and azimuthal angle, $\theta$ and $\phi$ throughout this paper. The quantity $\varphi^{i}$ depends on the direction of the motion of the quasiparticles. On the other hand, global phase $\chi_{0}$ is independent of the direction of the motion of the quasiparticles.

To discuss the physical meaning of these two phases more clearly, let us consider a trajectory of a quasiparticle injected from the superconductor to the interface. The quasiparticle does not always feel the same phase of the pair potential before and after the reflection.28.29, 30) We denote the phase felt by the quasiparticle before and after the reflection as $\bar{\varphi}_{1}$ and $\bar{\varphi}_{2}$, respectively, with $\bar{\varphi}_{1}=\varphi_{1}^{i}+\chi_{0}$ and $\bar{\varphi}_{2}=\varphi_{2}^{i}+\chi_{0}$, where $\varphi_{1}^{i}$ and $\varphi_{2}^{i}$ are the internal phases of the pair potentials. The condition of the ZES is determined by the phase increment felt by the quasiparticle, $\bar{\varphi}_{1}-\bar{\varphi}_{2}$. It should be remarked that this condition is determined by $\varphi_{1}^{i}-\varphi_{2}^{i}$ and is regardless of the choice of $\chi_{0}$. Consequently, we can verify the U(1)-gauge invariance of the condition of ZES.

In this paper, the effects of the spatial dependence of the pair potential have not been determined self-consistently. In anisotropic superconductors, the spatial dependence of the pair potentials is important for a quantitative discussion.54) For some cases a coexistence of pair potentials is expected.55 A coexistence of several pair potentials at the interface is expected to influence the tunneling conductance. Furthermore, the influence of the roughness of the surface on the conductance is also an interesting problem. $50,57,58$ 


\section{Acknowledgments}

We would like to thank Prof. A. Doenni for critical reading of our paper. One of the authors (Y.T.) is supported by a Grant-in-Aid for Scientific Research in Priority Areas, "Anomalous metallic states near the Mott transition," and "Nissan Science Foundation". The computation in this work has been done using the facilities of the Supercomputer Center, Institute for Solid State Physics, University of Tokyo.

[1] For recent reviews of heavy Fermion superconductivity, see e.g., M. Sigrist and K. Ueda, Rev. Mod. Phys. 63, 231 (1991); R. H. Heffner and M. R. Norman, Comments Cond. Mat. Phys. 17, 361 (1996), and references therein.

[2] H. Tou, Y. Kitaoka, K. Asayama, N. Kimura, Y. Ōnuki, E. Yamamoto, and K. Maezawa, Phys. Rev. Lett. 77, 1374 (1996).

[3] K. A. Park and Robert Joynt, Phys. Rev. Lett. 74, 4734 (1995); Phys. Rev. B 53, 12346 (1996).

[4] D.W. Hess, T.A. Tokuyasu, and J.A. Sauls, J. Phys. Condens. Matter 1, 8135 (1989).

[5] M. E. Zhitomirsky and Kazuo Ueda, Phys. Rev. B 53, 6591 (1996).

[6] K. Machida and M. Ozaki, Phys. Rev. Lett. 66, 3293 (1991); T. Ohmi and K.Machida, Phys. Rev. Lett. 71, 625 (1993); K. Machida, T. Ohmi, and M. Ozaki, J. Phys. Soc. Jpn. 62, 3216 (1993).

[7] K. Machida and T. Ohmi, J. Phys. Soc. Jpn. 65, 3456 (1996); T. Ohmi and K. Machida, J. Phys. Soc. Jpn. 65, 4018 (1996).

[8] K. Machida, T. Ohmi, and M. Ozaki, J. Phys. Soc. Jpn. 64, 1067 (1995).

[9] J. A. Sauls, J. Low Temp. Phys. 95, 153 (1994); Adv. Phys. 43, 143 (1994).

[10] A. Fledderjohann and P. J. Hirschfeld, Solid State Commun. 94, 163 (1995).

[11] M. R. Norman and P. J. Hirschfeld, Phys. Rev. B 53, 5706 (1996).

[12] M. J. Graf, S.-K. Yip, and J. A. Sauls, J. Low Temp. Phys. 102, 367 (1996).

[13] B. S. Shivaram, Y. H. Jeong, T. F. Rosenbaum, and D. G. Hinks, Phys. Rev. Lett. 56, 1078 (1986).

[14] B. Ellman, L. Taillefer, and M. Poirier, Phys. Rev. B 54, 9043 (1996).

[15] L. Taillefer, B. Ellman, B. Lussier, and M. Poirier, Physica B 230-232, 327 (1997)

[16] B. Lussier, B. Ellman, and L. Taillefer, Phys. Rev. Lett. 73, 3294 (1994); Phys. Rev. 53, 5145 (1996).

[17] H. Suderow, J. P. Brison, A. Huxley, and J. Flouquet, J. Low Temp. Phys. 108, 11 (1997).

[18] Y. Maeno, H. Hashimoto, K. Yoshida, S. Nishizaki, T. Fujita, J. G. Bednorz, and F. Lichtenberg, Nature (London) 372, 532 (1994).

[19] Y. Maeno and K. Yoshida, in Proceedings of the 21st International Conference on Low Temperature Physics, Pragua, 1996 [Czech. J. Phys. 46, 3097 (1996), Suppl. S6.]

[20] K. Yoshida, Y. Maeno, S. Nishizaki, and T. Fujita, Physica C 263, 519 (1996).

[21] Y. Maeno, S. Nishizaki, K. Yoshida, S. Ikeda, and T. Fujita, J. Low. Temp. Phys. 105, 1577 (1996).

[22] K. Ishida, Y. Kitaoka, K. Asayama, S. Ikeda S. Nishizaki, Y. Maeno, K. Yoshida, and T. Fujita, Phys. Rev. B 56, R505 (1997)

[23] T. M. Rice and M. Sigrist, J. Phys. Condens. Matter 7, 643 (1995).

[24] I. I. Mazin and David J. Singh, Phys. Rev. Lett. 79, 733 (1997).

[25] M. Sigrist and M. E. Zhitomirsky, J. Phys. Soc. Jpn. 65, 3452 (1996).

[26] K. Machida, M. Ozaki, and T. Ohmi, J. Phys. Soc. Jpn. 65, 3720 (1996).

[27] D. J. van Harlingen, Rev. Mod. Phys. 67, 515 (1995). 
[28] Y. Tanaka and S. Kashiwaya, Phys. Rev. Lett. 74, 3451 (1995); Phys. Rev. B 53, 9371 (1996).

[29] S. Kashiwaya, Y. Tanaka, H. Takashima, Y. Koyanagi, and K. Kajimura, Phys. Rev. B 51, 1350 (1995).

[30] S. Kashiwaya, Y. Tanaka, M. Koyanagi, and K.Kajimura, Phys. Rev. B 53, 2667 (1996); Jap. J. Appl. Phys. 34, 4555 (1995); J. Phys. Chem. Solids. 56, 1721 (1995).

[31] C. Bruder, Phys. Rev. B 41, 4017 (1990).

[32] A. F. Andreev, Zh. Eksp. Teor. Fiz. 46, 1823 (1964) [Sov. Phys. JETP 19, 1228 (1964)].

[33] G. E. Blonder, M. Tinkham, and T. M. Klapwijk, Phys. Rev. B 25, 4515 (1982).

[34] C. R. Hu, Phys. Rev. Lett. 72, 1526 (1994).

[35] J. Geerk, X. X. Xi, and G. Linker, Z. Phys. B 73, 329 (1988).

[36] J. Lesueur, J., L. H. Greene, W. L. Feldmann, and A. Inam, Physica C 191, 325 (1992).

[37] L. Alff, H. Takashima, S. Kashiwaya, N. Terada, H. Ihara, Y. Tanaka, M. Koyanagi, and K. Kajimura, Phys. Rev. B 55, R14757 (1997).

[38] S. Ueno, S. Kashiwaya, N. Terada, M. Koyanagi, Y. Tanaka, and K. Kajimura, to be published in J. of Phys. Chem. Solids.

[39] For recent reviews, see e.g., H.v. Löhneysen, Physica B 218, 148 (1996); ibid. 197, 551 (1994).

[40] G. Goll, C. Bruder, and H.v. Löhneysen, Physica B 206-207, 609 (1995); Phys. Rev. B 52, 6801 (1995).

[41] A. Nowack, A. Heinz, F. Oster, D. Wohlleben, G. Güntherodt, Z. Fisk, and A. Menovsky, Phys. Rev. B 36, 2436 (1987).

[42] G. Goll, H.v. Löhneysen, I.K. Yanson, and L. Taillefer, Phys. Rev. Lett. 70, 2008 (1993).

[43] Y. DeWilde, J. Heil, A. G. M. Jansen, P. Wyder, R. Deltour, W. Assmus, A. Menovsky, W. Sun, and L. Taillefer, Phys. Rev. Lett. 72, 2278 (1994).

[44] Yu. G. Naidyuk, H.v. Löhneysen, G. Goll, C. Paschke, I. K. Yanson, and A. A. Menovsky, Physica B 218, 161 (1996).

[45] L. J. Bichholtz and G. Zwicknagl, Phys. Rev. B, 23, 5788 (1981).

[46] J. Hara and K. Nagai, Prog. Theor. Phys. 74, 3451 (1986).

[47] G. Deutscher and P. Nozières, Phys. Rev. B 50, 13557 (1994).

[48] J. Kurkijärvi and D. Rainer, in Helium Three, edited by W. P. Halperin and L. P. Pitaevskii (Elsevier, Amsterdam, 1990).

[49] M. Yamashiro, Y. Tanaka, Y. Tanuma, and S. Kashiwaya, Physica C 293, 239 (1997).

[50] Y. Tanaka and S. Kashiwaya, in Qunatum Coherence and Decoherence, edited by K. Fujikawa and Y.A. Ono (North-Holland, 1996).

[51] M. Yamashiro, Y. Tanaka, and S. Kashiwaya, Phys. Rev. B 56, 7847 (1997).

[52] C. Honerkamp and M. Sigrist, cond-mat[9706199].

[53] Y. Tanaka and S. Kashiwaya, Phys. Rev. B 53, R11957 (1996); ibid. 56, 892 (1997).

[54] Yu. S. Barash, A. A. Svidzinsky, and H. Burkhardt, Phys. Rev. B 55, 15282 (1997).

[55] M. Matsumoto and H. Shiba, J. Phys. Soc. Jpn. 64, 3384 (1995); 64, 4867 (1995); 65, 2194 (1996).

[56] K. Yamada, Y. Nagato, S. Higashitani, and K. Nagai, J. Phys. Soc. Jpn. 65, 1540 (1996).

[57] Y. Nagato, M. Yamamoto, and K. Nagai, unpublished

[58] Y. Tanuma, Y. Tanaka, M. Yamashiro, and S. Kashiwaya, Physica C 293, 234 (1997); Phys. Rev. B 57, 7997 (1998). 
Table I. The region on the Fermi surface contributing to ZES for $\mathrm{UPt}_{3}$

\begin{tabular}{ccc}
\hline & $z$-y plane interface & $x$ - $y$ plane interface \\
\hline $\mathrm{A}_{1 u}, \mathrm{~A}_{2 u}$ & none & $0 \leq \phi \leq 2 \pi, 0 \leq \theta \leq \pi / 2$ \\
$\mathrm{E}_{1 u}, \mathrm{E}_{2 u}$ & $\phi= \pm \pi / 4$ & $0 \leq \phi \leq 2 \pi, 0 \leq \theta \leq \pi / 2$ \\
$\mathrm{E}_{1 g}$ & $\phi=0$ & $0 \leq \phi \leq 2 \pi, 0 \leq \theta \leq \pi / 2$ \\
\hline
\end{tabular}

Table II. The region on the Fermi surface contributing to ZES for $\mathrm{Sr}_{2} \mathrm{RuO}_{4}$

\begin{tabular}{lcc}
\hline & $z$-y plane interface & $x$ - $y$ plane interface \\
\hline $\mathrm{E}_{u}(1), \mathrm{E}_{u}(\mathrm{U} 1)$ & $-\pi / 4 \leq \phi \leq \pi / 4$ & none \\
$\mathrm{E}_{u}(2), \mathrm{E}_{u}(\mathrm{U} 2)$ & $\phi=0$ & none \\
\hline
\end{tabular}

Fig. 1. (a): Schematic illustration of the reflection and the transmission process of the quasiparticle at the interface of the junction with $z-y$ plane interface and (b): $x-y$ plane interface. The $\theta$ and $\phi$ are the polar angle and azimuthal angle, respectively.

Fig. 2. Normalized conductance for (a) the $\mathrm{A}_{1 u}$ state and (b) the $\mathrm{A}_{2 u}$ state when the interface is perpendicular to the $x$ - and the $z$-axes, as indicated in the figure. Effective barrier parameters are a: $Z=0.1, \mathrm{~b}: Z=1$ and c: $Z=5$. 
Fig. 3. Normalized conductance for (a) the $\mathrm{E}_{1 u}$ state and (b) the $\mathrm{E}_{2 u}$ state when the interface is perpendicular to the $x$ - and the $z$-axes, as indicated in the figure. Effective barrier parameters are a: $Z=0.1, \mathrm{~b}: Z=1$ and c: $Z=5$.

Fig. 4. Normalized conductance for the $\mathrm{E}_{1 g}$ state when the interface is perpendicular to the $x$ - and the $z$-axes, as indicated in the figure. Effective barrier parameters are a: $Z=0.1, \mathrm{~b}: Z=1$ and $\mathrm{c}: Z=5$.

Fig. 5. Normalized conductance for (a) the $\mathrm{E}_{u}(\mathrm{U} 1)$ state and (b) the $\mathrm{E}_{u}(\mathrm{U} 2)$ state when the interface is perpendicular to the $x$ - and the $z$-axes, as indicated in the figure. Effective barrier ( $\delta$-function model) parameters are $\mathrm{a}: Z=0.1, \mathrm{~b}: Z=1$ and $\mathrm{c}: Z=5$.

Fig. 6. Normalized conductance for (a) the $\mathrm{E}_{u}(1)$ state and (b) the $\mathrm{E}_{u}(2)$ state when the insulator has a finite thickness $d_{i}$ for $\kappa=0.5$ with a: $\lambda_{0} d_{i}=0.1, \mathrm{~b}: \lambda_{0} d_{i}=1$ and $\mathrm{c}: \lambda_{0} d_{i}=5$. 
(a)

(ab)

electron

Alu ( $z-y$ plane interfaco lane interface

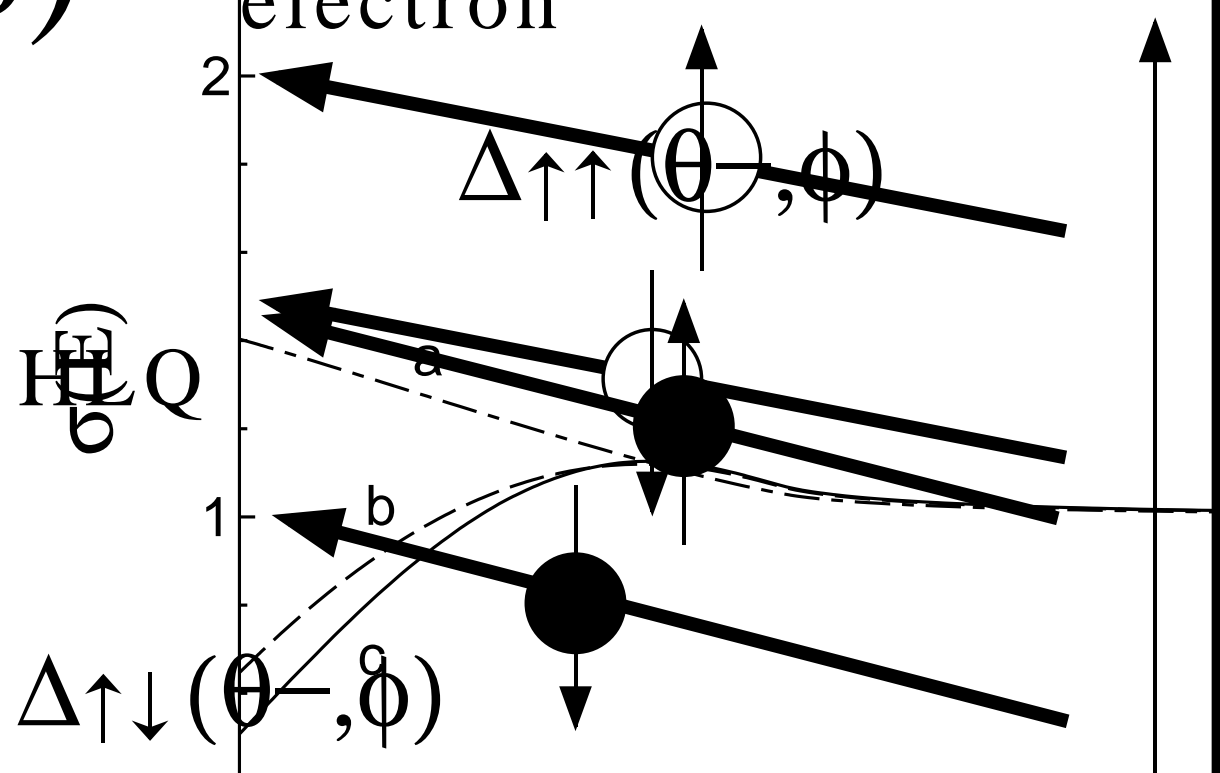

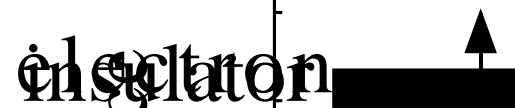

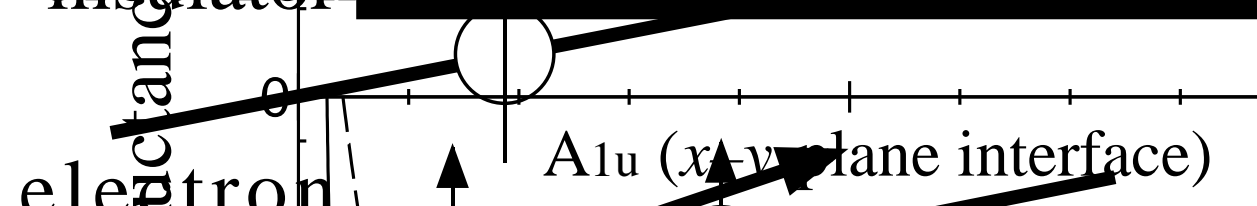

eleggtron

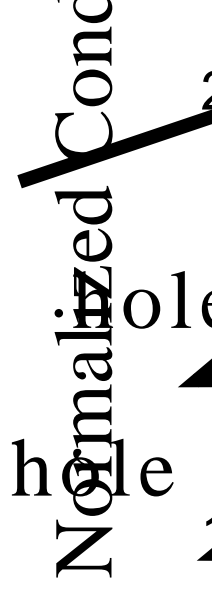




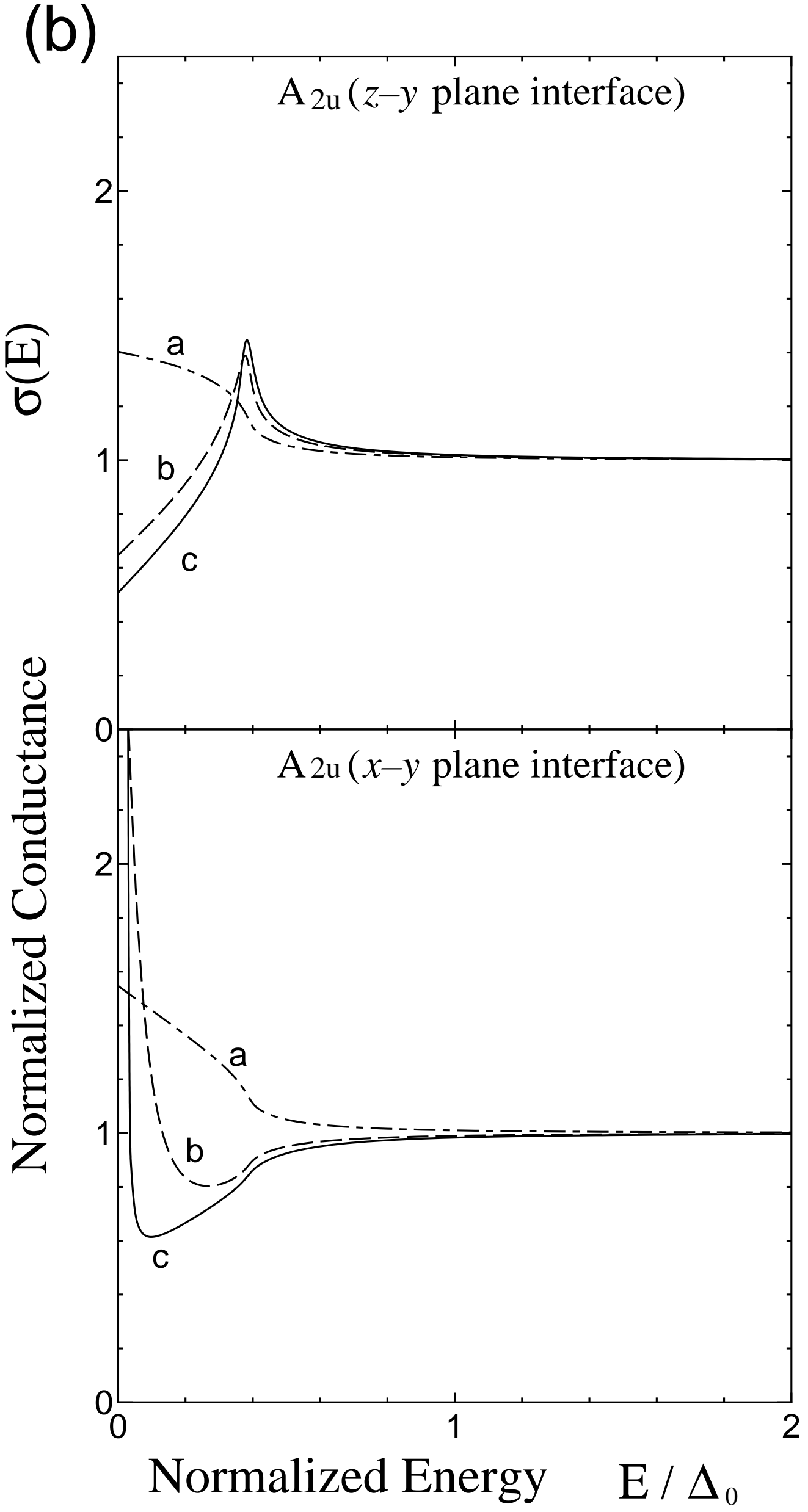




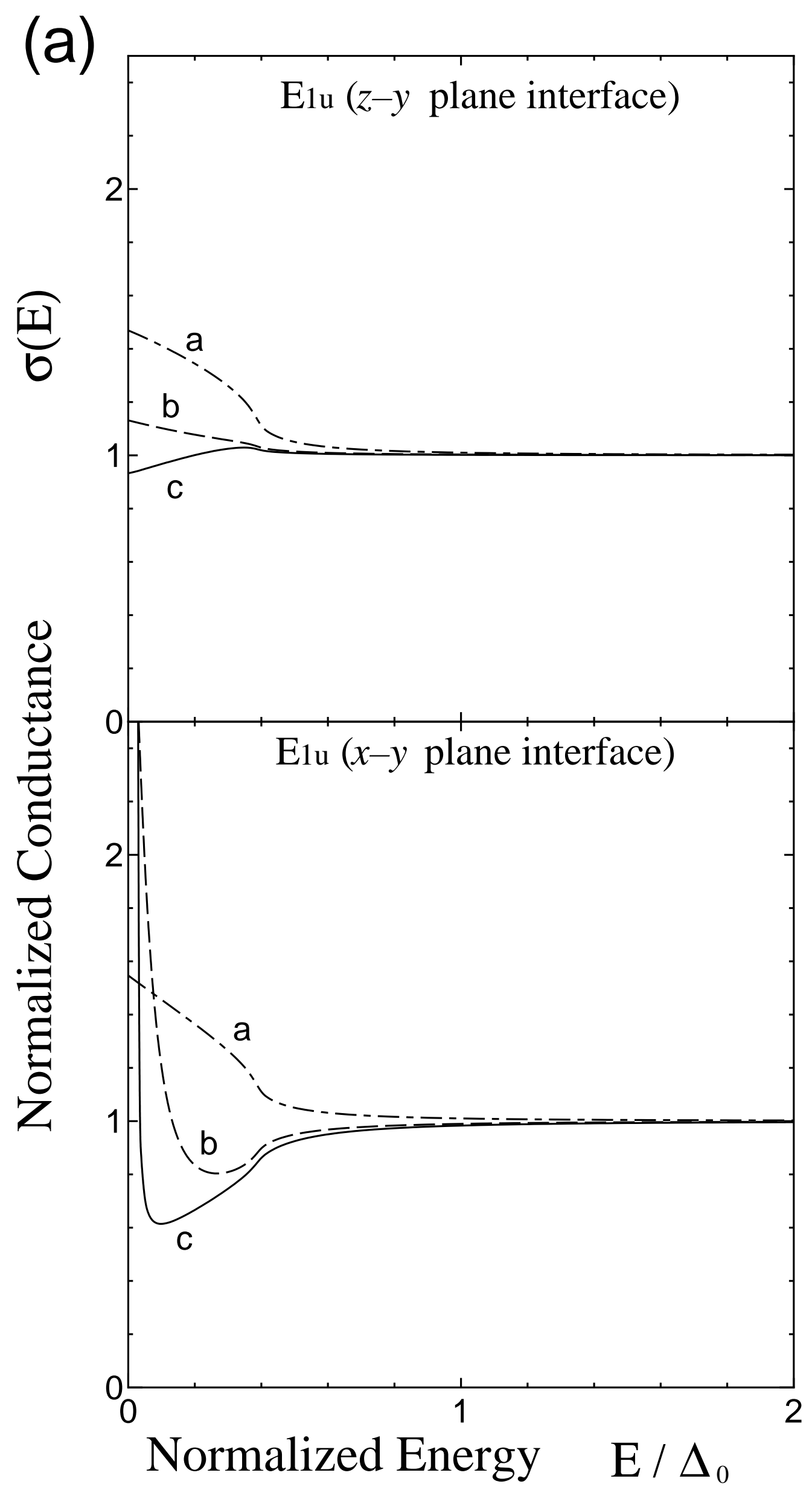


(b)

(b)

E2u (z-y plane interface)

四

2

(1)

c

e

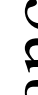

$$
\text { . }
$$

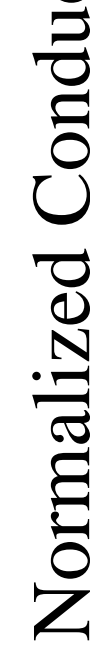

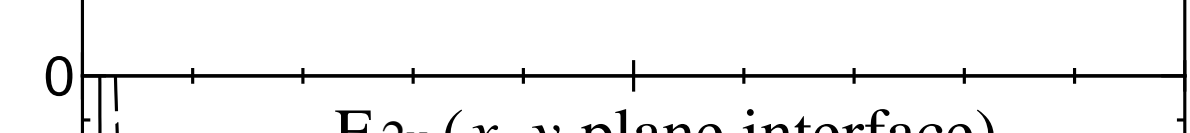




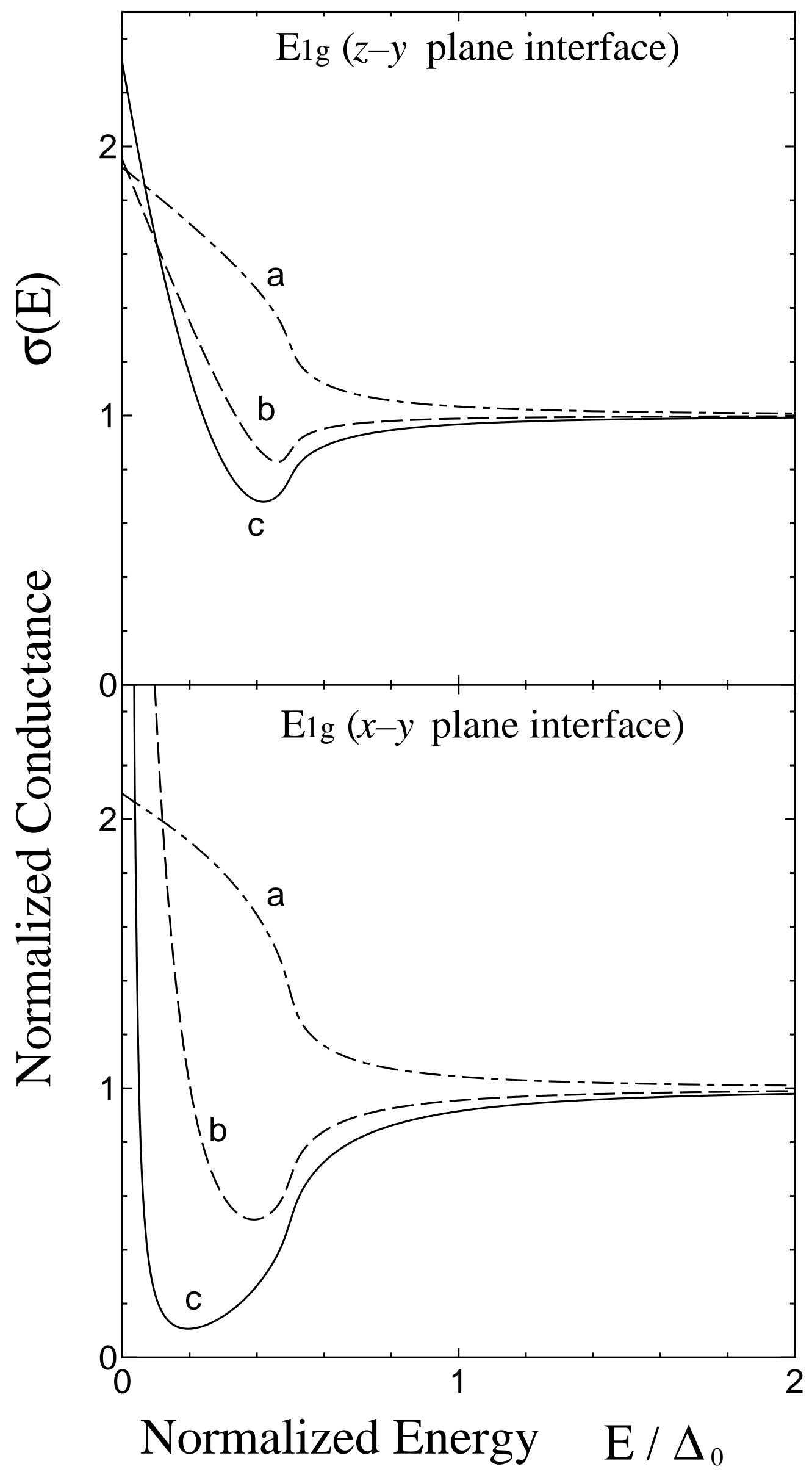




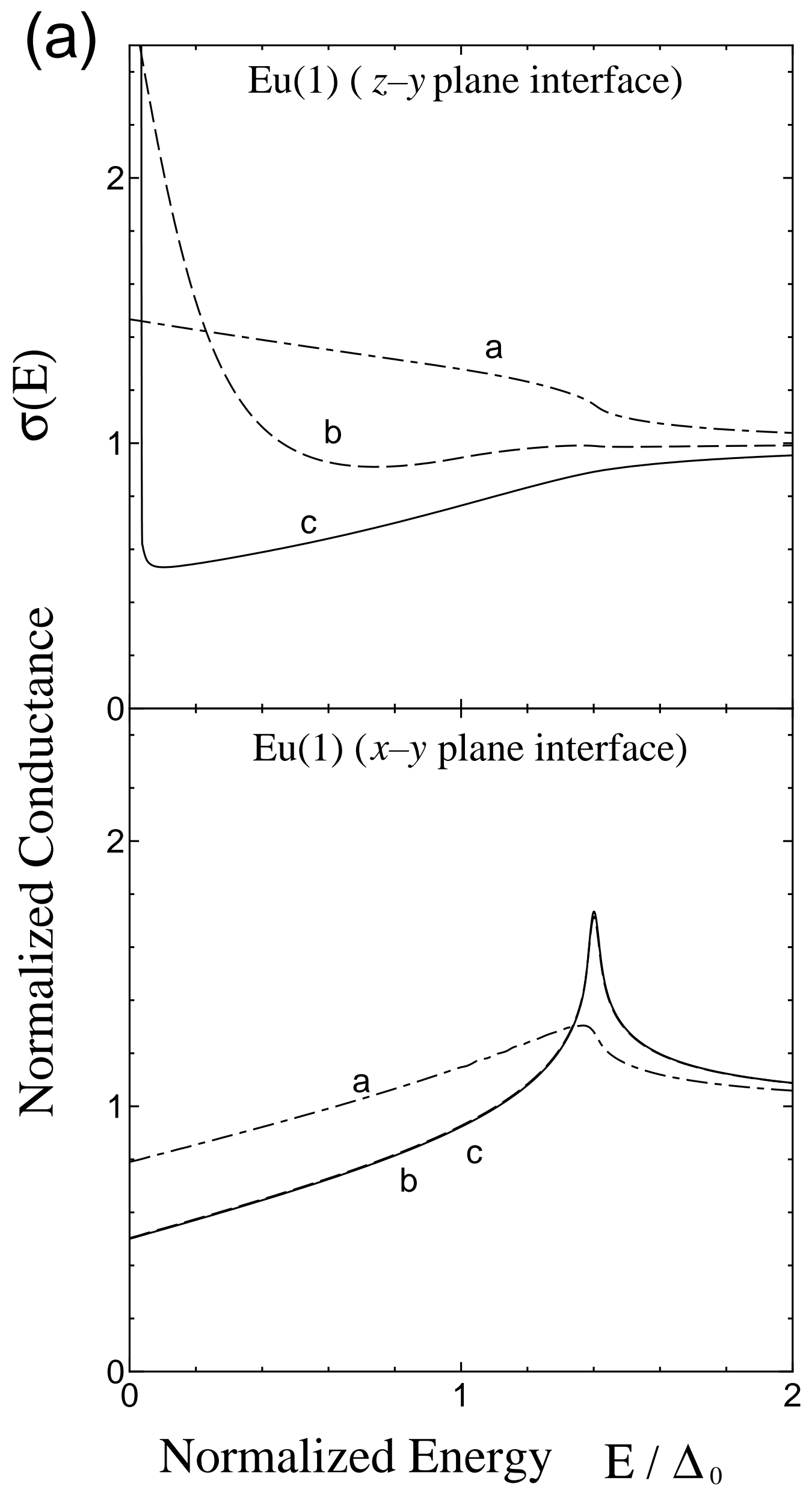




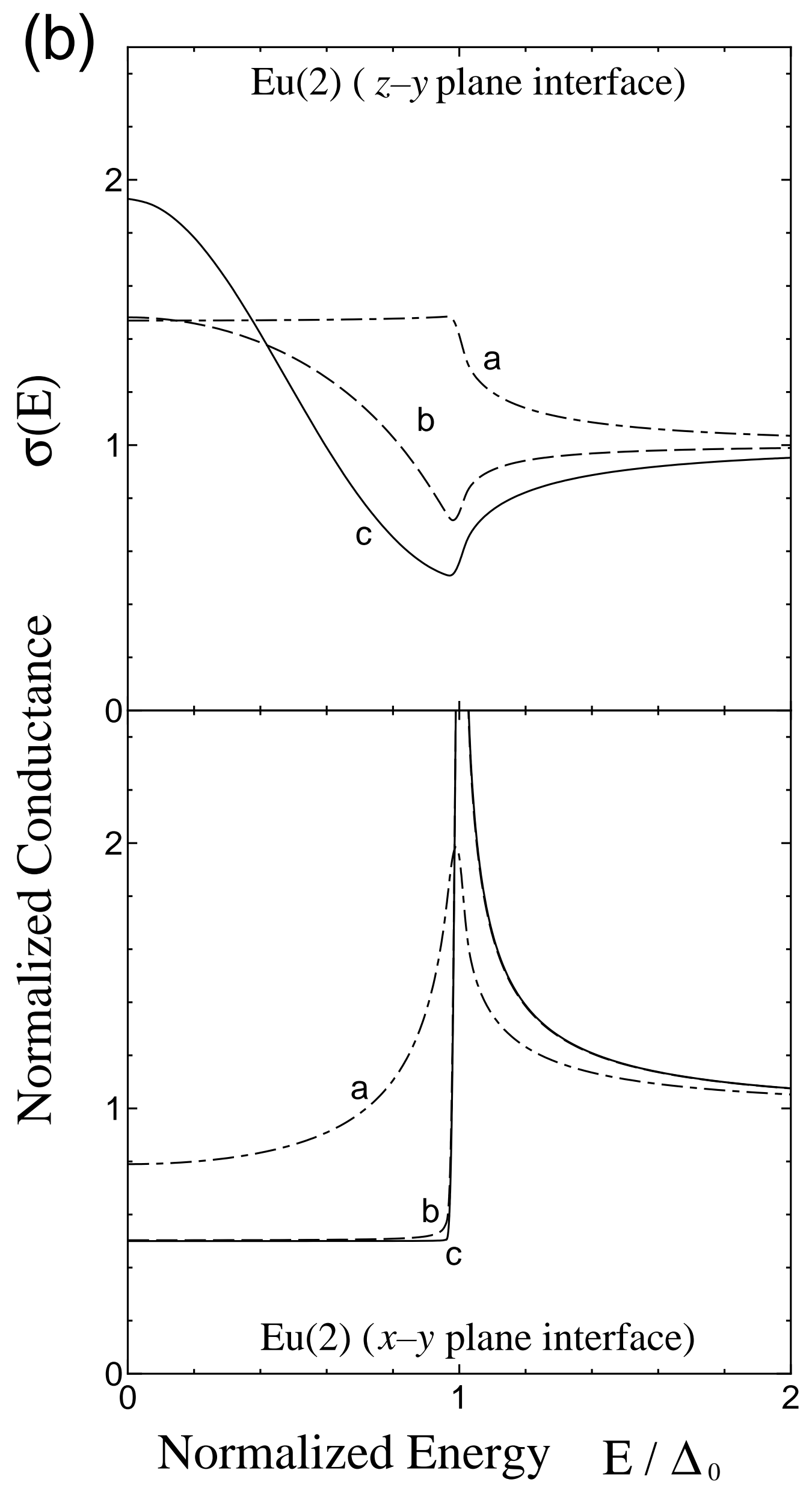




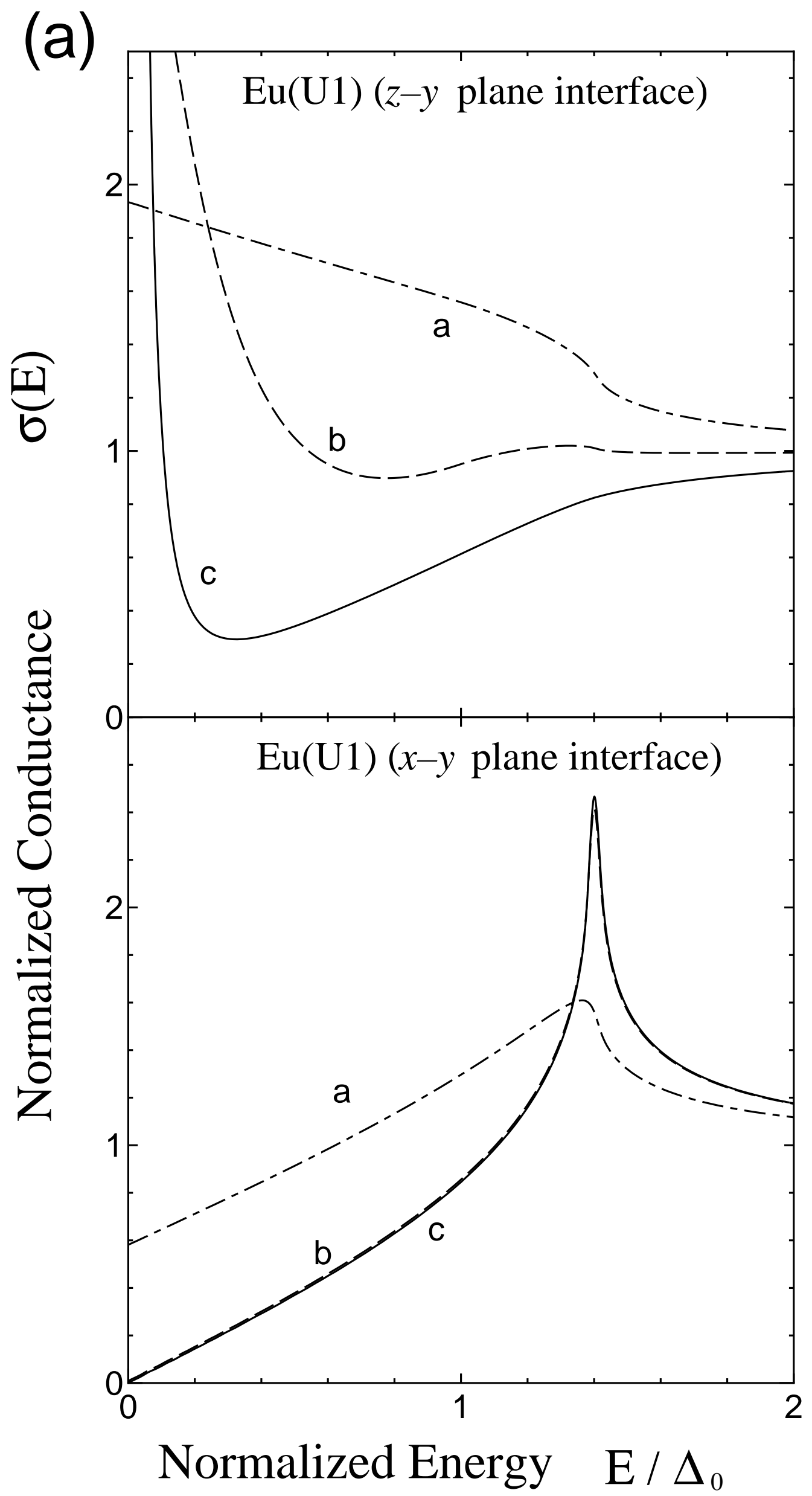




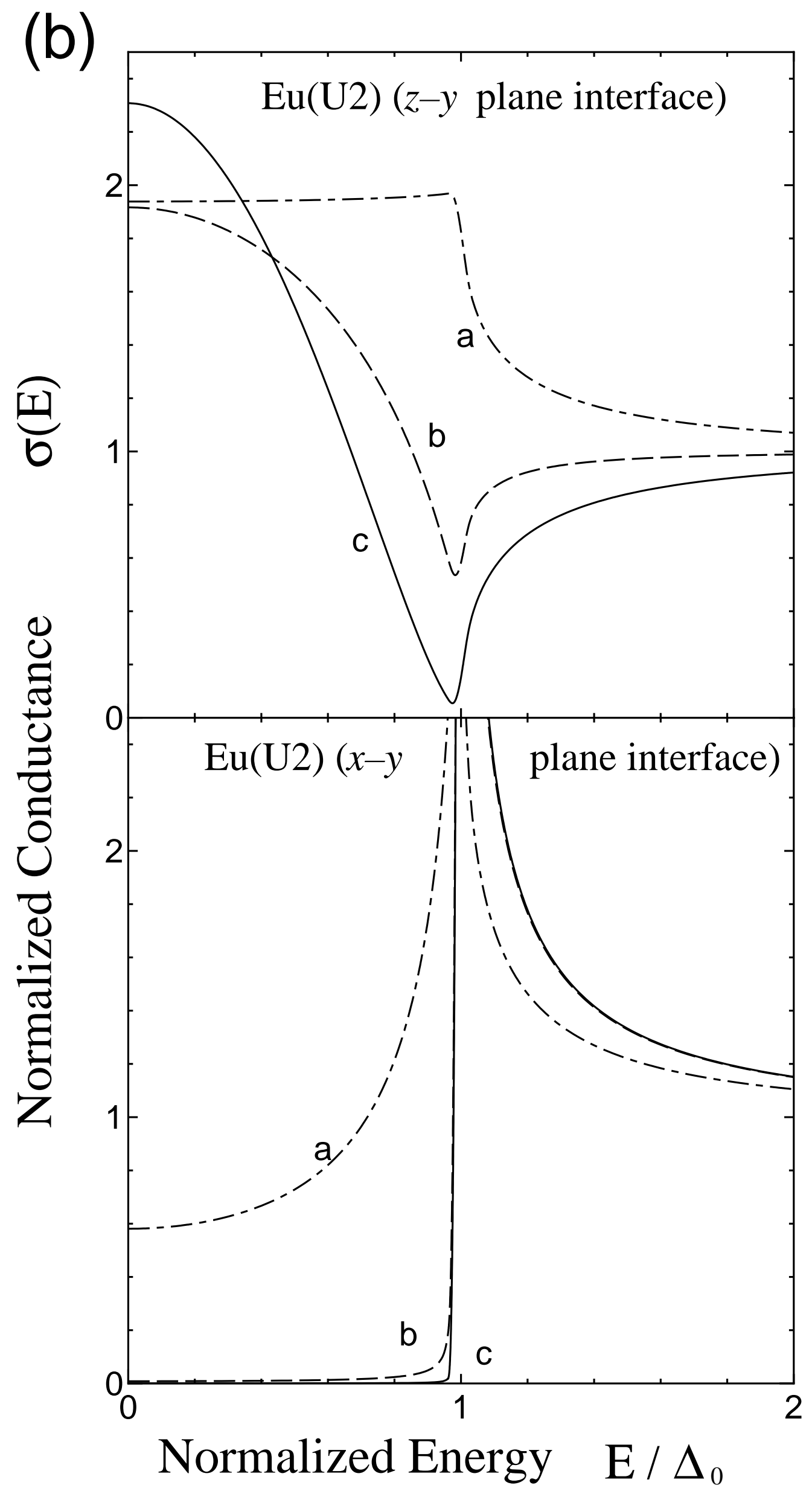

